\title{
Successful cryopreservation of spermatozoa following orchidectomy for testicular torsion in a patient with previous contralateral torsion of testis
}

\begin{abstract}
A 25-year-old patient with a previously missed left testicular torsion and subsequent left testicular atrophy presented with torsion of the right testicle. He underwent orchidectomy as an emergency and post procedure he underwent successful cryopreservation of sperm after 2 days.
\end{abstract}

\author{
Volume 6 Issue I - 2018

\begin{abstract}
James K Worsfold, Soumendra N Datta
Department of Urology, Colchester University Hospital NHS Foundation Trust, United Kingdom
\end{abstract}

\begin{abstract}
Correspondence: Dr. Soumendra Datta, Consultant Urological Surgeon, Department of Urology, Colchester Hospital, Turner Road, Colchester, CO4 5JL, United Kingdom, Emailsam.datta@colchesterhospital.nhs.uk
\end{abstract}

Received: November 15, 2017 | Published: January 18, 2018

\section{Background}

Testicular torsion has an incidence of 1 in 4000 of patients less than 25 years old. ${ }^{1}$ Untreated cases will result in testicular necrosis and atrophy. The greater the time taken for the patient to present, and the higher the patients' age, are both factors associated with an increased risk of requiring an orchidectomy. ${ }^{2}$ Fertility following testicular torsion has been shown to be reduced. ${ }^{3}$ The reduction in fertility is documented in patients following orchidectomy with a contralateral viable testicle. In this case the contralateral testicle was atrophic following previous misdiagnosed torsion. Patients with severe testicular atrophy have undergone successful microdissection of sperm, in one study up to $56 \%$ of patients with non-obstructive testicular atrophy had successful microdissection of sperm. These cases were more successful in patients with Kleinefelter's syndrome as opposed to other causes of testicular atrophy. ${ }^{4}$ Cryopreservation of sperm through masturbation rather than microdissection has been used for patients with testicular malignancy undergoing elective orchidectomy, both pre and post-operatively. ${ }^{5}$ There is a documented case of a patient undergoing orchidopexy for torsion with ischaemic testicular injury and a single testis having a successful sperm cryopreservation post operatively. ${ }^{6}$ There are no documented cases of patients underwent successful sperm cryopreservation following orchidectomy for testicular torsion. There has been some research into storage of sperm in seminal vesicles which may explain the mechanism for successful cryopreservation following orchidectomy however studies have produced mixed results., ${ }^{78}$ The success of seminal vesicle aspiration in fertile volunteers was affected by the number of days of abstinence which is a factor which cannot be changed in an emergency presentation.?

\section{Case presentation}

A 25 year old gentleman presented to the acute urology team with sudden onset sharp right sided testicular pain. The pain was severe, constant and had been increasing in severity over the preceding 36 hours. There were no clinical features of systemic infection, no fevers, no dysuria and no penile discharge. He had experienced left sided testicular pain 10 years previously in Lithuania; this lasted for 1 week and subsequently improved without treatment. On examination the left sided testicle was atrophic. The right scrotum was swollen, warm and erythematous, with a normal lie clinically. Given the clinical suspicion of torsion he underwent emergency scrotal exploration +/- bilateral orchidectomy. Intra-operatively the right testicle was torted and clinically infarcted. The testicle remained non-viable following treatment with $100 \%$ oxygen and warm gauze wrapping for 10 minutes. The left testicle was severely atrophic with minimal parenchyma - features likely due to a previous left sided torsion. A right orchidectomy and left sided orchidopexy were completed. Histology of the right testis and cord showed ischaemic necrosis secondary to testicular torsion. He was referred for urgent semen cryopreservation post operatively. He attended for cryopreservation two days after the orchidectomy and was able to provide a semen sample with viable sperm for IVF. He has subsequently started testosterone replacement therapy which is being managed by his GP. The sperm freeze record results are shown below. Day 2 post orchidectomy the semen analysis showed a sperm density of 2 million per ml. In 2010 the WHO defined a normal sperm density as over 15 million per ml. ${ }^{9}$ Histology results from the right testicle revealed ischaemic necrosis, confirming testicular torsion as the cause of acute pain. 8 weeks post orchidectomy the patient had a raised FSH and LH and a low testosterone level, showing that the atrophic left testis is non-functioning.

\section{Investigations}

(Table $1 \& 2$ ).

Table I Patient cryopreservation results Day 2 and Day 3 post right orchidectomy

\begin{tabular}{lllll}
\hline Freeze date & $\begin{array}{l}\text { Density } \\
\left(\times 10^{6} / \mathrm{ml}\right)\end{array}$ & $\begin{array}{l}\text { Progressive } \\
\%\end{array}$ & $\begin{array}{l}\text { Abnormal } \\
\text { Forms (\%) }\end{array}$ & $\begin{array}{l}\text { No. of Vials } \\
\text { Frozen }\end{array}$ \\
\hline Day 2 post op & 2 & $48 \%$ & $5 \%$ & 2 \\
Day 3 post op & $\begin{array}{l}\text { Occasional } \\
\text { Motile Sperm }\end{array}$ & - & 2 \\
\hline
\end{tabular}

\section{Discussion}

Testicular torsion is one of the more common urological emergencies. ${ }^{1}$ Timely diagnosis and a high index of suspicion with 
early testicular exploration improve outcomes and reduce the potential for testicular necrosis and subsequent orchidectomy. ${ }^{2}$ Patients with single testis or previous testicular atrophy are at greatest risk of infertility following orchidectomy. In this case, prompt referral for cryopreservation of sperm following emergency scrotal exploration and orchidectomy resulted in a successful cryopreservation. Greater public awareness of the potential consequences of sudden onset testicular pain would allow for earlier presentation and prompt diagnosis, thus reducing the risk of orchidectomy. ${ }^{2}$ In cases with delayed presentation and previous contralateral testicular atrophy or orchidectomy, consideration of urgent semen cryopreservation post operatively gives the patients the option of future fertility treatment using the patient's own semen, despite a lack of viable testicular tissue. Given the results above, the rapid time following orchidectomy to presentation for cryopreservation is important, with abstinence being required following surgery before the initial cryopreservation. The 8 weeks post operative results for this patient show that the left sided atrophic testis is non-functioning. We can therefore conclude that the successful cryopreservation has come from sperm produced from the right testicle prior to orchidectomy. After the testicle was removed the spermatozoa present in either the seminal vesicles, ${ }^{7}$ or the vas deferens ${ }^{10}{ }^{10}$ allowed for successful cryopreservation to take place. This also explains why the second sample obtained on day 3 post-operatively contains only occasional motile sperm, as once this remaining reservoir of spermatozoa has been utilised in the initial sample obtained, no further spermatozoa production is taking place.

Table 2 Patient results 8 weeks post orchidectomy with normal values. ${ }^{11-13}$

\begin{tabular}{lll}
\hline & $\mathbf{8}$ Weeks post orchidectomy & Normal values \\
\hline FSH & 105.6 & $\mathrm{I} .0-18.0 \mathrm{IU} / \mathrm{L}$ \\
$\mathrm{LH}$ & 45.8 & $\mathrm{I} .8-8.6 \mathrm{IU} / \mathrm{L}$ \\
Testosterone & 0.58 & $8.3-32.9 \mathrm{nmol} / \mathrm{L}$ \\
\hline
\end{tabular}

\section{Conclusion}

Cryopreservation of sperm following emergency orchidectomy is possible, even with a contralateral non functioning testis, provided it takes place in the immediate post operative period. More awareness of potential cryopreservation treatment will allow a greater proportion of patients to benefit from preserved fertility following emergency orchidectomy. Greater education of the public to ensure early presentation with acute testicular torsion will allow early scrotal exploration and bilateral orchidopexy, preventing the requirement for orchidectomy and safeguarding against contralateral torsion in the future.

\section{Funding}

None.

\section{Acknowledgements}

None.

\section{Conflicts of interest}

None.

\section{References}

1. Tomlinson MJ, Kohut TL, Hopkisson JF, et al. Routine sperm banking for testicular cancer patients should be performed both before and after orchidectomy. Journal of Clinical Urology. 2013;6(3):171-176.

2. Mansbach JM, Forbed P, Peters C. Testicular torsion and risk factors for orchiectomy, Archives of Paediatric and Adolescent Medicine. 2005;159(12):1167-1171.

3. Bartsch G, Frank S, Marberger H, et al. Testicular Torsion: Late Results with Special Regard to Fertility and Endocrine Function. $J$ Urol. 1980;124(3):375-378.

4. Bryson CF, Ramasamy, Sheehan M, et al. Severe Testicular Atrophy does not Affect the Success of Microdissection Testicular sperm Extraction. $J$ Urol. 2014;191(1):175-178.

5. Sibert L, Rives N, Rey D, et al. Semen Cryopreservation after Orchidectomy in men with testicular cancer. BJU Int. 1999;84(9): 1038-1042.

6. Woodruff DY, Horwitz G, Weigel J, et al. Fertility preservation following torsion and severe ischemic injury of a solitary testis. Ferti Steril. 2010;94(1):552-555.

7. Jarrow J. Seminal Vescicle Aspiration of Fertile Men. J Urol. 1996;156(3):1005-1007.

8. Ponig BF, Roberts JA. Seminal vescicles as organs of sperm storage urology. 1978;11(4):384-385.

9. Cooper TG, Noonan E, Von Eckardstein S, et al. World Health Organisation reference values for human semen characteristics. Hum Reprod Update. 2010;16(3):231-245.

10. Peirce EJ, Moore HD, Leigh CM, et al. Studies on sperm storage in the vas deferens of the spinifex hopping mouse (Notomys Alexis). Reproduction. 2003;125(2):233-240.

11. Follicle stimulating hormone (FSH), Serum. Mayo clinic laboratories.

12. Testosterone, total, bioavailable and free (TTFB), Serum. Mayo Clinic Laboratories.

13. Lutenizing hormone (LH), Serum, Mayo clinic laboratories. 